\title{
Cordycepin ameliorates synaptic dysfunction and dendrite morphology impairments induced by cerebral ischemia via adenosine A1 receptors in vitro and in vivo
}

\author{
Zhao-Hui Chen ${ }^{1}$, Ying-Jie Shang ${ }^{1}$, Si-Yi Zhuang ${ }^{1}$, Jun-Ni Huang ${ }^{1}$, Bao-Yan Wu \\ Wen-Xiao $\mathrm{Liu}^{1}$, and Chu-Hua $\mathrm{Li}^{1}$ \\ ${ }^{1}$ South China Normal University
}

June 15, 2020

\begin{abstract}
Background and aims: Cordycepin has been proved to have neuroprotection and to improve learning and memory in cerebral ischemia. However, the potential mechanisms are unclear so far. Plasticy of synaptic structure and synaptic function are considered as the neural mechanisms of learning and memory. Therefore, we investigated the effects of cordycepin on dendritic morphology and synaptic function in cerebral ischemic models. Experimental Approach: The impact of cordycepin was studied using oxygen glucose deprivation (OGD) and global cerebral ischemia (GCI) models. Synaptic transmission and behavioral long-term potentiation (LTP) were investigated with electrophysiological recordings. Dendritic morphology was assessed by Golgi staining. The densities of adenosine A1 and A2A receptors (A1R and A2AR) were evaluated with western blots and immunofluorescence. Key Results: Cordycepin alleviated the ischemia-induced damages of dendritic morphology and behavioral LTP in hippocampal CA1 area, improved the learning and memory abilities and up-regulated the expression of A1R but not $\mathrm{A} 2 \mathrm{AR}$ in hippocampus of GCI rats. In the in vitro experiments, cordycepin pre-perfusion could reduce the hippocampal slices injury and synaptic transmission impairment induced by OGD, improved adenosine content and reduced the expression of A1R but did not alter A2AR. Furthermore, the protection of cordycepin on synaptic transmission against OGD was eliminated by using the antagonist of A1R instead of A2AR. Conclusion and Implications: These findings indicated that cordycepin alleviated synaptic dysfunction and dendritic injury in vivo and in vitro ischemic models by modulating A1R, which may be the neural mechanisms of cordycepin to improve learning and memory in cerebral ischemic animals.
\end{abstract}

\section{Hosted file}

Manuscript.doc available at https://authorea.com/users/333570/articles/459737-cordycepinameliorates-synaptic-dysfunction-and-dendrite-morphology-impairments-induced-by-

cerebral-ischemia-via-adenosine-a1-receptors-in-vitro-and-in-vivo 
A

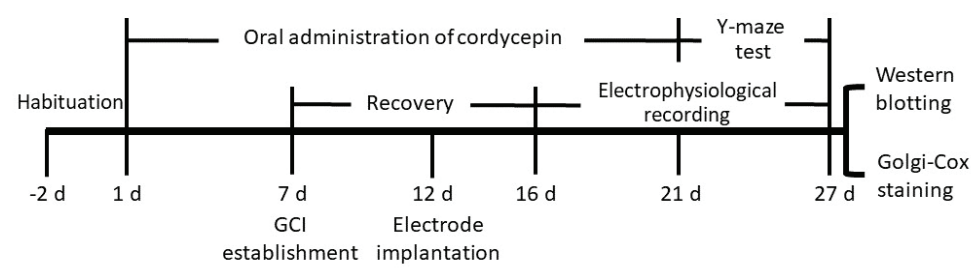

In vivo experiment

B

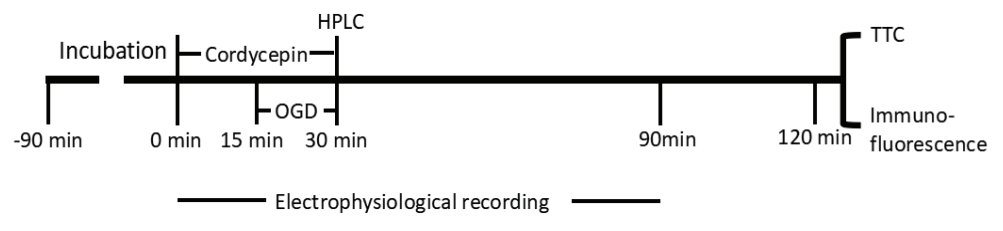

In vitro experiment

Figure 1 
A

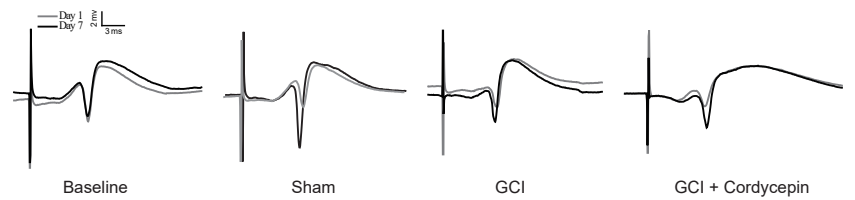

B

C
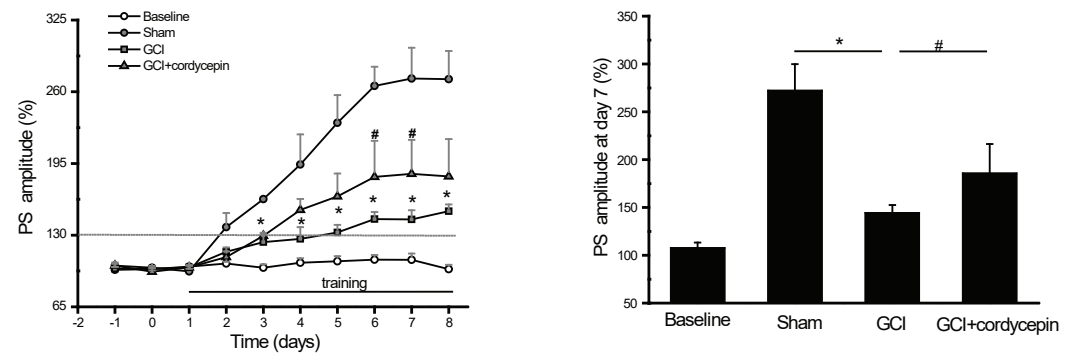

D

E
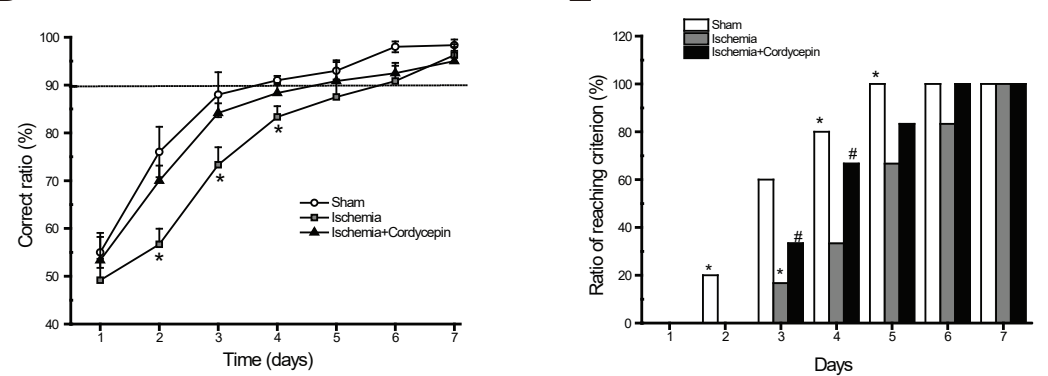

Figure 2 
A

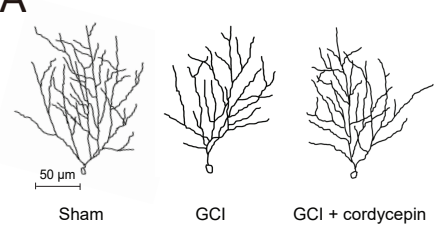

C

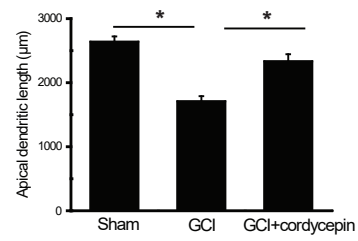

E

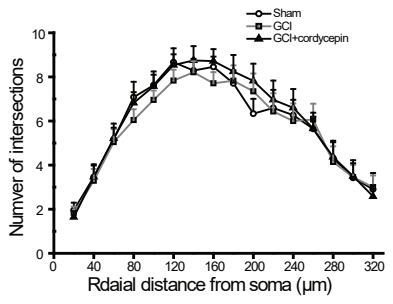

G
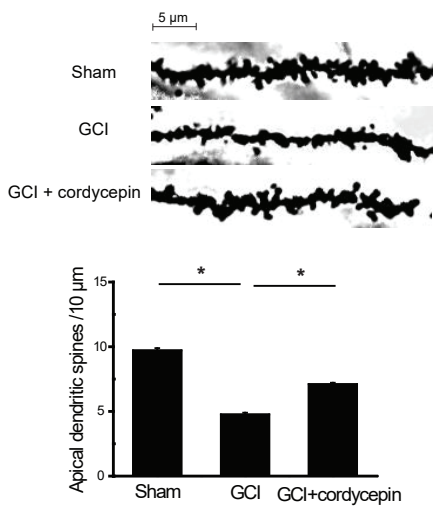

B

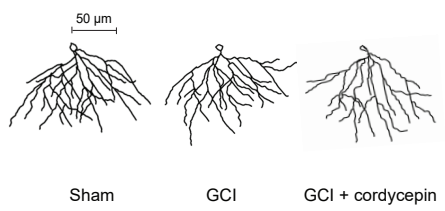

D

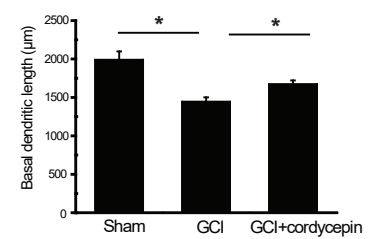

F

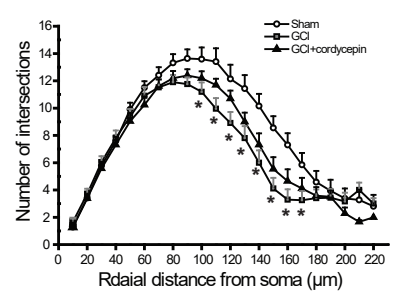

$\mathrm{H}$
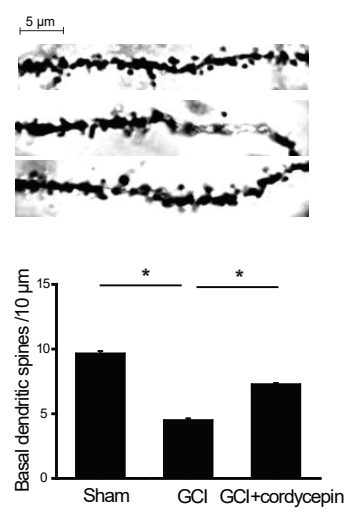

Figure 3 
A
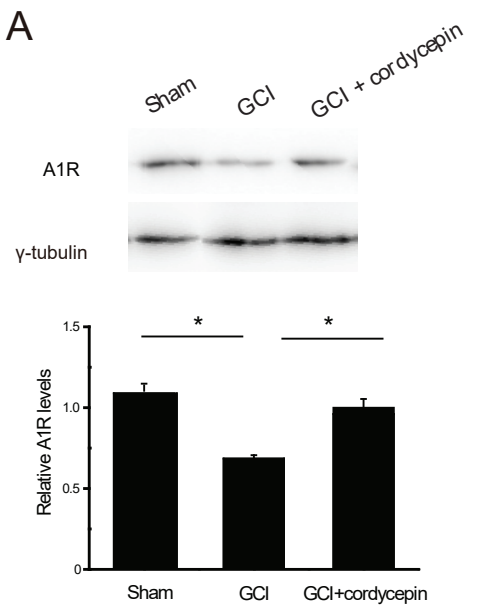

B
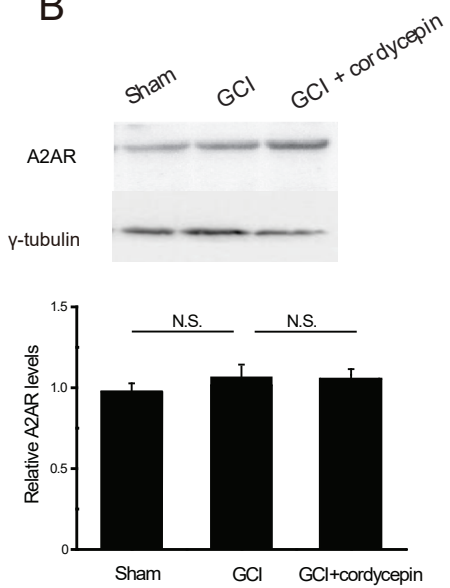

Figure 4 
A
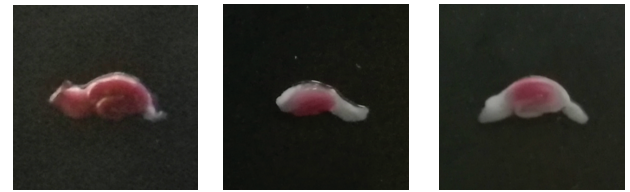

$15 \mathrm{~min}$

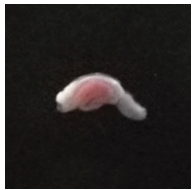

Control

$10 \min$

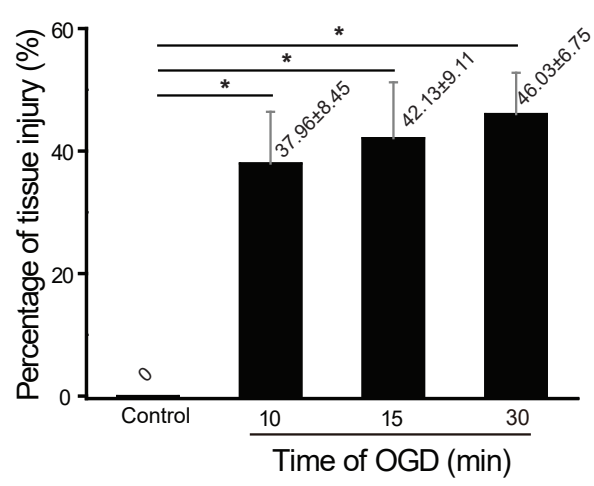

B
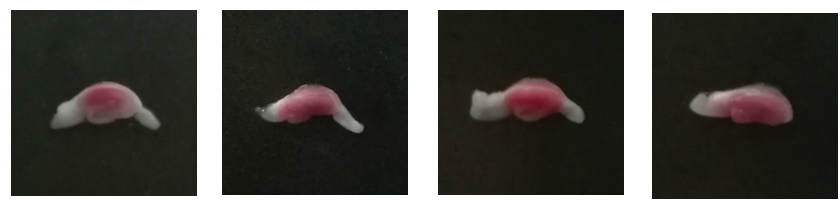

OGD 15 min

10

20

Cordycepin (ug/mL) + OGD 15min

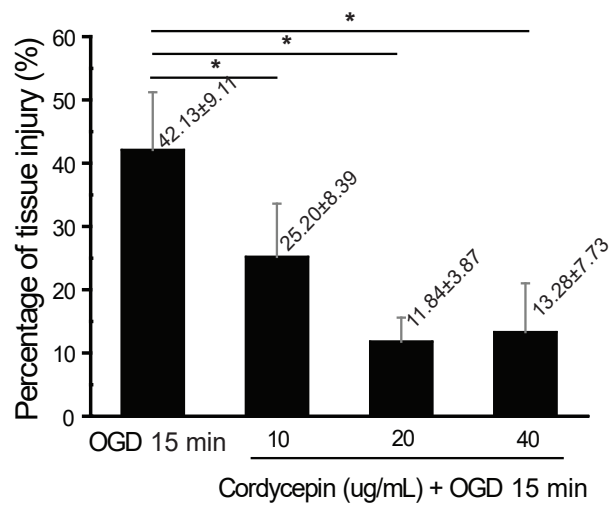

Figure 5 


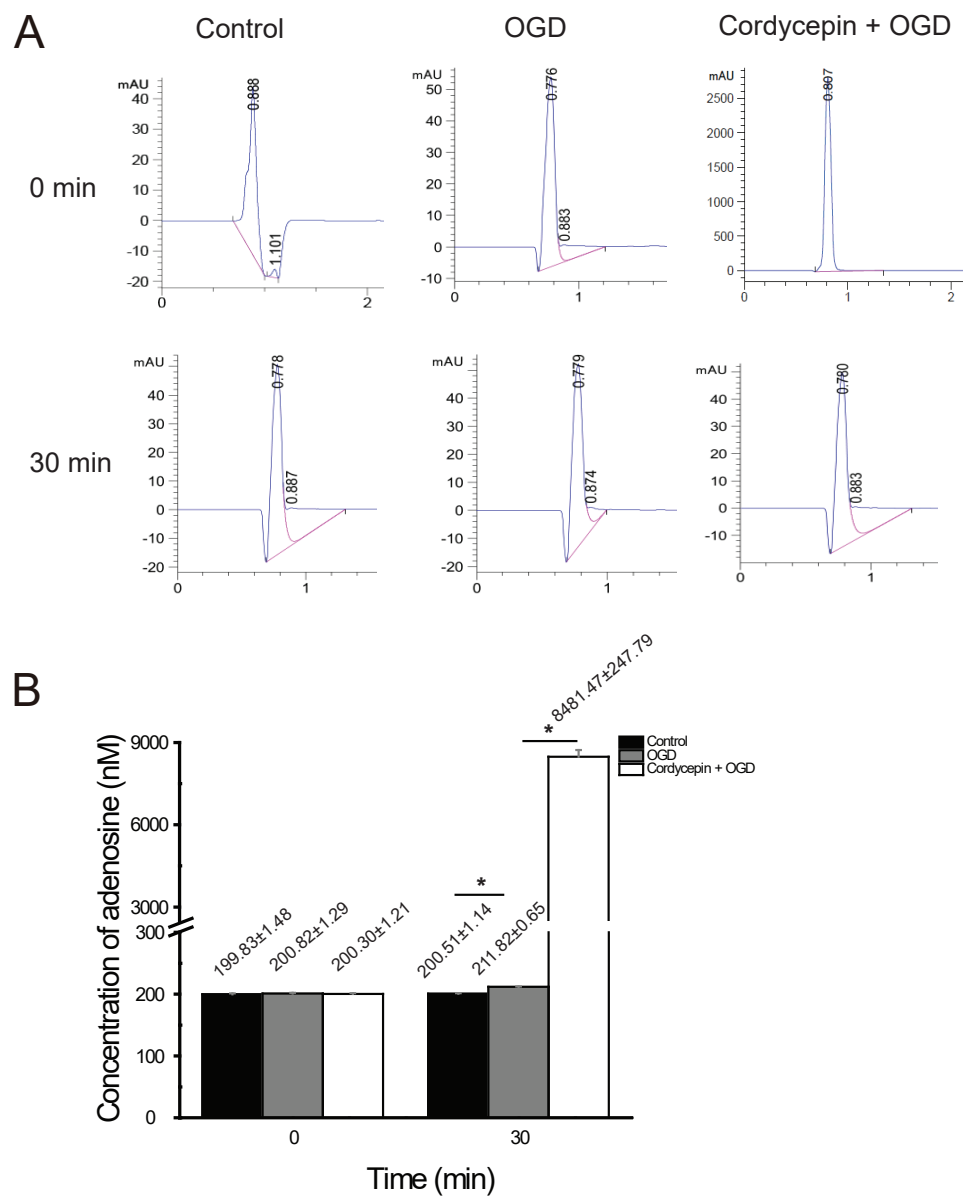

Figure 6 
A
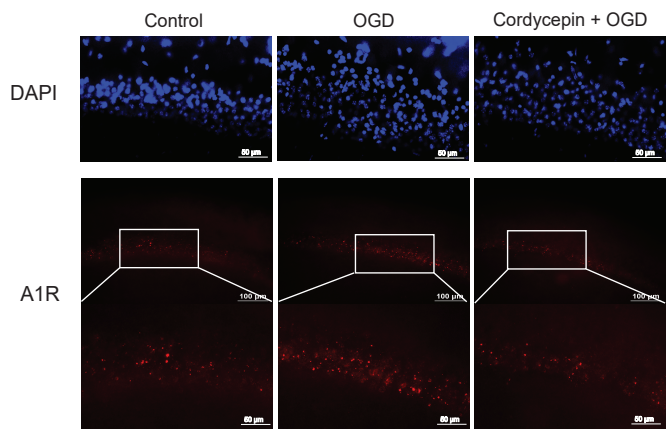

C

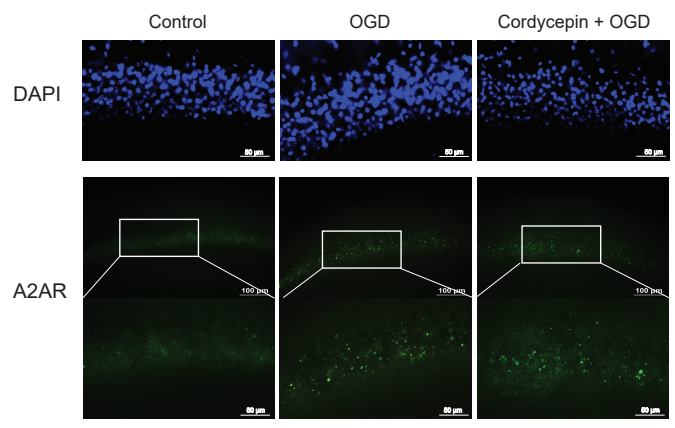

B

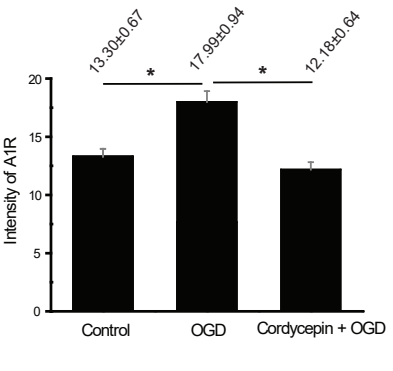

D

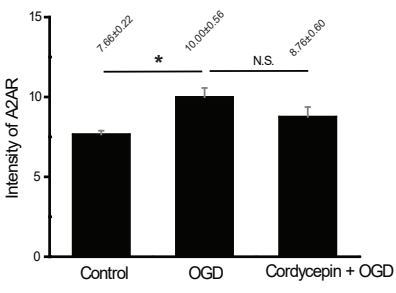

Figure 7 


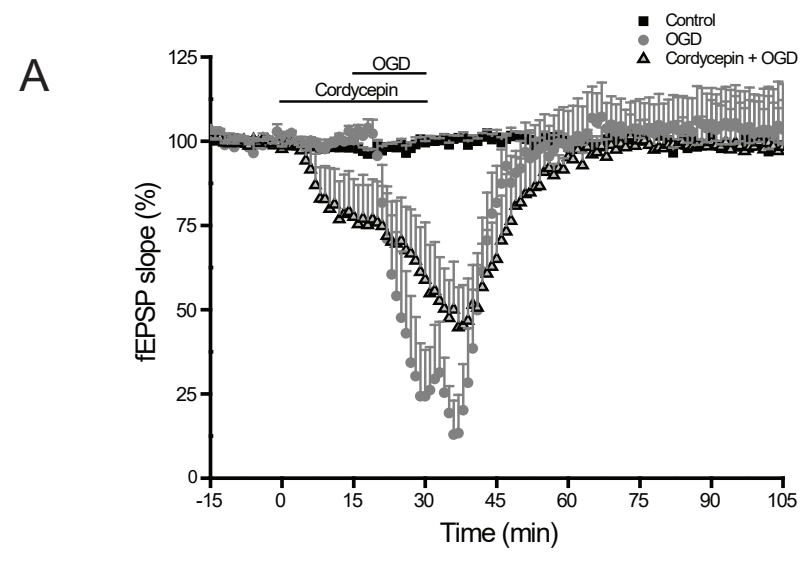

B
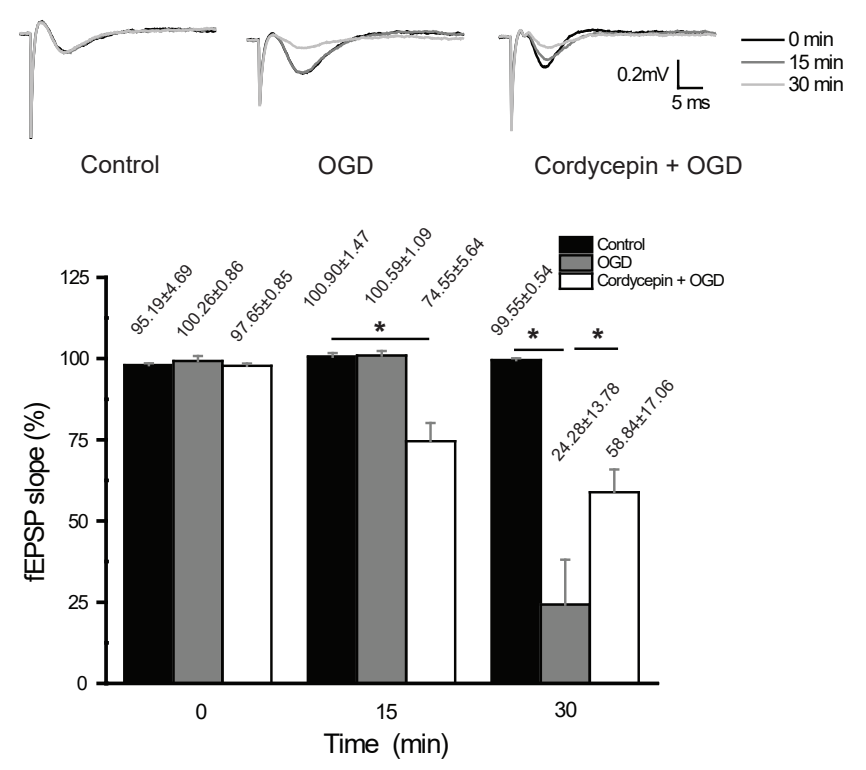

Figure 8 
A

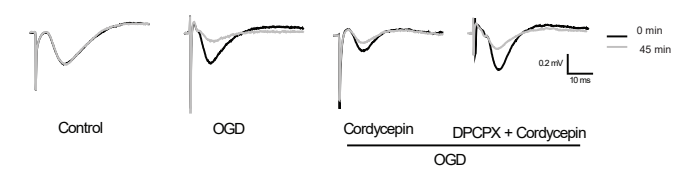

B

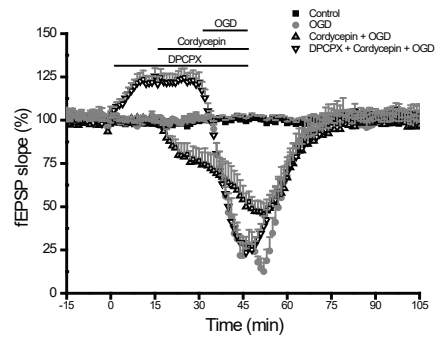

C

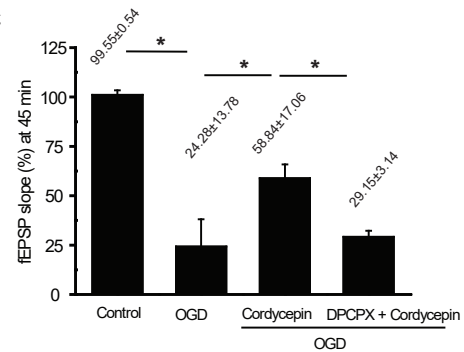

D

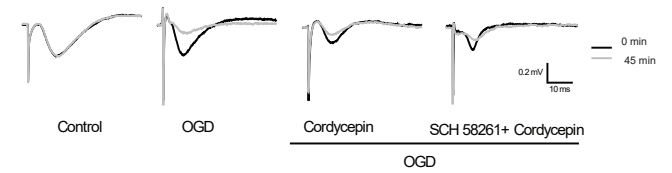

E
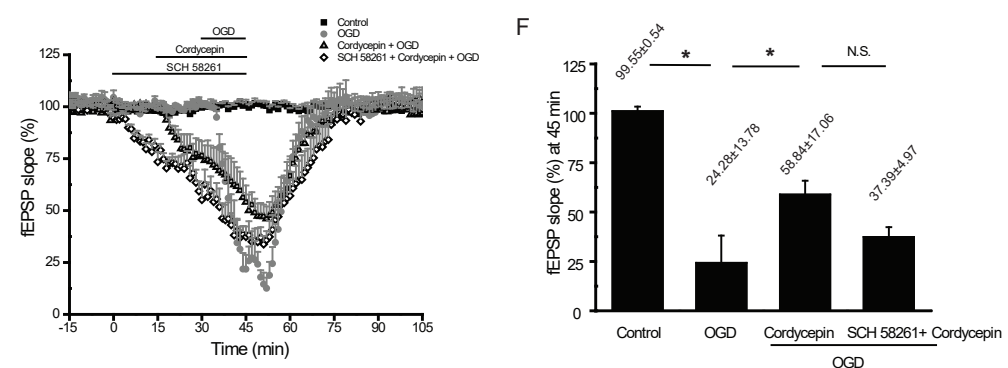

Figure 9 